\title{
Patching Physics and Chemistry Together
}

\author{
Department of Philosophy, Logic and Scientific Method \\ London School of Economics \\ Houghton St. London WC2A 2AE, United Kingdom
}

and

\author{
Center for Junior Research Fellows \\ PO Box M682 \\ University of Konstanz \\ D-78457 Konstanz, Germany
}

Word Count: 4439

To be presented at PSA 2004 in Austin, Texas and to appear in the accompanying Philosophy of Science Supplement.

\begin{abstract}
The "usual story" regarding molecular chemistry is that it is roughly an application of quantum mechanics. That is to say, quantum mechanics supplies everything necessary and sufficient, both ontologically and epistemologically to reduce molecular chemistry to quantum mechanics. This is a reductive story, to be sure, but a key explanatory element of molecular chemistry, namely molecular structure, is absent from the quantum realm. On the other hand, typical characterizations of emergence, such as the unpredictability or inexplicability of molecular structure based on quantum mechanics do not characterize the relationship between molecular chemistry and quantum mechanics well either. A different scheme for characterizing reduction and emergence is proposed that accommodates the relationship between quantum mechanics and molecular chemistry and some initial objections to the scheme are considered.
\end{abstract}


Even within physical science, reduction between different levels of explanation is problematic-indeed, it is almost always so.

Sir Michael Berry

\section{Introduction}

How are physics and chemistry related? The typical answer to this question in chemistry textbooks as well as much literature in philosophy and the sciences is reductionist, much in the spirit of Paul Dirac's famous quote:

The underlying physical laws necessary for the mathematical theory of a large part of physics and the whole of chemistry are thus completely known, and the difficulty is only that the exact application of these laws lead to equations much too complicated to be soluble (Dirac 1929, p. 714).

There are, of course, some powerful philosophical justifications for this kind of answer. For example, mereological dependence-where properties of wholes depend in some way on properties of their parts-looks to indicate that quantum physics supplies the "parts" for for the "wholes"-molecules-of chemistry. In other words, quantum physics provides the base from which the properties of chemical molecules arises.

Another justification for this reductionist perspective comes from the completeness of physics (CoP). Here, we can distinguish two forms. In the epistemic form, CoPe, physics aims for a complete description of physical phenomena. Since descriptions of chemical phenomena crucially involve elements from physics, physics descriptions ought to yield chemistry descriptions (modulo derivational complications referred to by Dirac). In the ontological form, CoPo, physics involves fundamental laws of all matter. Since, chemical phenomena crucially involve elements from physics, the laws and properties of physics ought to yield chemical laws and properties (modulo derivational complications).

The usual story for this reductionist relationship between chemistry and physics, runs roughly as follows (Hendry 1998): Chemistry should be a completely quantum mechanical affair as the first principles underlying chemistry are to be found in the domain of quantum mechanics. Therefore, in principle we can get chemistry from physics by some form of deduction, implication or derivation. For instance, in quantum chemistry, one first specifies the fundamental physical interactions (electromagnetic, strong- and weak-nuclear, etc.), then enumerates the relevant particles and their properties (nucleon, electron, charge, mass, etc.). Next, one lists the pairwise interactions among the particles. Finally, one writes down the kinetic and potential energy operators and adds them to get the system Hamiltonian (an expression for the total energy of the system). With the Hamiltonian in hand, one then proceeds to derive the properties and behaviors of the chemical system in question.

But, as the usual story continues, this program is mathematically intractable. We can neither write the fundamental Hamiltonian down nor do we have the computational resources to carry the program through (Dirac's complications). No worries, though, as there are a number of approximation methods affording the construction of tractable, if inexact, Hamiltonians (e.g., associating electronic states with a "fixed-nucleus" Hamiltonian, or treating molecular vibrations in 
the small oscillation limit). The approximate equations derived from these procedures are then taken to stand in for the unobtainable first principles equations. This story has been called the "Proxy defense" by Robin Hendry (1998). In the epistemic version of this story, the less fundamental theory is deducible from more fundamental theory, while in the ontological version, the less fundamental phenomena are deducible or derivable from the more fundamental phenomena plus the fundamental laws.

In this essay, I want to demonstrate how molecular chemistry, in particular the feature of molecular structure, challenges this usual reductionist story. However, I will also point out how molecular structure challenges a quite popular formulation of emergence as well. I will end by sketching an alternative way of reformulating such notions as reduction and emergence, relating this alternative account to the feature of molecular structure and defending it against some objections.

\section{Challenging the Usual Story: Molecular Structure}

Molecular structure or shape plays a crucial causal role in chemistry. It dominates the interpretations of the calculations and experiments of chemists. More importantly, it has empirical and practical import. Differentiation of isotopes is based on molecular structure as well as the explanation for properties like acidity. Molecular chirality (handedness of molecules) led to disaster in thalidomidebased treatments in the 1960s, because one species of handedness was harmful while the other was beneficial. Molecular structure and chirality are crucial for the explanation of optical activity, where particular materials, so-called optical isomers, rotate plane-polarized light passing through them (Wolley 1976, p. 32). They are invoked in typical descriptions and interpretations of single biomolecule spectroscopy (Weiss 1999) and in the nanomechanical properties of molecules (Gimzewski and Joachim 1999; Smith et al. 1999). And they play a crucial role in understanding DNA, various diseases and medications (e.g., DeCamp 1989; Avertisov et al. 1991). Furthermore, molecular structure is important for chemical and biological self-assembly (e.g., Lehn 2002; Whitesides and Grzybowski 2002).

But there is a problem here because the "true" molecular Hamiltonian at the level of quantum mechanics-could we actually write it down-would not exhibit any features corresponding to molecular structure and, hence, would provide no basis for distinguishing isotopes or describing optical activity and other phenomena dependent on molecular structure. Furthermore, the full molecular Schrödinger equation would have too many symmetries, e.g., nuclear permutation and rotational symmetries missing from real molecules. However, the explanation of such phenomena are counted as great successes for the so-called molecular structure hypothesis.

So it appears that we have successful structural explanations at the level of molecular chemistry but no firm quantum foundation! As Guy Woolley puts it, "Systematic application of quantum mechanics to a molecule does not lead...to the usual, and undoubtably essentially correct, description of chemical phenomena that is obtained from orthodox quantum chemistry" (Woolley 1976, p. 31). The usual story-the proxy defense-comes up short as the structural models used by chemists as well as the empirical data do not represent solutions to any potential quantum mechanical first principles equations. It appears that in order to handle molecular structure and its implications, "new concepts which cannot be inferred from the underlying fundamental laws [of quantum mechanics] are required" (Woolley 1976, p. 32).

How does molecular structure arise in quantum chemistry? The concept of molecular structure was first precisely defined in 1927 through the Born-Oppenheimer "approximation" 
(1927). The basic idea is to assume the nuclear mass is very much larger than the electron mass, then treat the nuclei as if they are (almost) stationary with respect to electronic motions. Mathematically this corresponds to an asymptotic series expansion in powers of , = (electron mass/nuclear mass) ${ }^{1 / 4}$, where, goes to zero as the nuclear mass is allowed to become large with respect to the electron mass. In this limit the correlations among nuclei and electrons as well as nuclear permutation and rotational symmetries are broken. ${ }^{1}$

The reason for the quotation marks around 'approximation' above is that the BornOppenheimer "approximation" or procedure, is not simply a mathematical expansion in series form, where only terms to small order in , are kept. It literally replaces the basic quantum description with a new description generated by the limit , 60 . This replacement corresponds to a change in the algebra of observables needed for the description of molecular phenomena-a change yielding chirality (and other features of molecular structure) as a classical observable (e.g., Primas 1983, pp. 335-341; Amann 1993). The Born-Oppenheimer approach amounts to a change in topology-i.e. a change in the mathematical elements modeling physical phenomena-as well as a change in ontology-including fundamental physical elements absent in quantum description; in the case of molecular chemistry, the new ontological elements are structures absent from quantum mechanics.

Now we can put the challenge of molecular structure for reduction very succinctly: Neither the topology nor the ontology appropriate to molecular structure can be derived from or found in quantum mechanics alone. Hence, an empirically and explanatorily important structure in molecular chemistry looks to be missing from quantum mechanics.

\section{Challenging Typical Emergence Accounts}

The standard account of emergence fares no better, however. Often, emergent properties are characterized as unpredictable in terms of a "more fundamental" theory as in Jageowan Kim's characterization of the standard version of property emergence (1999, pp. 19-22). Given the situation in $\S 2$, molecular structure indeed is not predictable from quantum mechanics, the more fundamental theory, alone. However, it turns out that molecular structure is predictable. For example, given the relevant elements of quantum mechanics (electrons, nucleons, etc., their associated algebra of observables and laws) and the contingent context provided by the BornOppenheimer procedure, i.e. the new topology and ontology associated with the limit , 60 , one can derive the emergent property of molecular structure (e.g., Primas 1998; Bishop and Atmanspacher submitted). Although quantum mechanics does play a necessary role in such a derivation or "prediction" of the new emergent property, the conditions supplied by the contingent context are crucial to the derivation.

Similarly, emergent properties often are characterized as unexplainable in terms of a "more fundamental" theory (see Kim 1999, pp. 19-22). Given quantum mechanics alone, molecular structure is unexplainable on covering-law, causal-mechanical and other traditional forms of explanation. That is to say, molecular structure is unexplainable in terms of derivations from the fundamental governing equations of quantum mechanics. However, it also turns out that molecular structure can be given an explanation. Again, given the relevant elements of quantum mechanics and

${ }^{1} \mathrm{~A}$ similar, and slightly more accurate, result can be achieved in the "adiabatic approximation" in which the electrons are considered to move much faster than the nuclear frame of a molecule. 
the contingent context provided by the Born-Oppenheimer procedure, one can give a quite rigorous explanation of the appearance of molecular structure (e.g., Primas 1998; Bishop and Atmanspacher submitted). As before, quantum mechanics does play a necessary role in such an explanation, but the conditions supplied by the contingent context are crucial to the success of the explanation.

Other oft cited characterizations of emergent properties-such as that they arise at higher levels out of properties and laws characterizing the entities and properties at a lower levels or that they have novel causal properties irreducible to the causal efficacy of lower-level properties-can be analyzed with respect to molecular structure in a similar fashion. This approach, only sketched abstractly here, deploys a powerful tool known as asymptotic expansions (see Friedrichs 1955; Dingle 1973; Berry 1994; Batterman 2002). In order to formulate such expansions, a reference state, representing essential features of the context, has to be specified in the original state space of the fundamental or lower-level theory. If the expansion is singular, as is the case for the BornOppenheimer procedure, it is not uniformly convergent in the original topology of the fundamental description as an appropriate parameter tends to some limit (e.g., as , 6 0). This discontinuous limiting behavior indicates the need for a change of topology. The crucial step, then, is to identify a new topology which regularizes the expansion such that it converges uniformly. This leads to a new contextual topology of the state space associated with novel properties not defined in the original state space under the original topology, and which is associated with ontological elements also not found at the level of the fundamental or lower-level theory.

It is worth emphasizing again that the new context, and its associated topology and ontology are never given by the original theory and its associated topology and ontology. Rather the new context is always a contingent matter; that is to say, it is tailored to particular situations. Clearly, features irrelevant in a particular context may be highly relevant in another. It is also worth pointing out that, although the approach outlined in this section provides a clear path for how the topologies and ontologies of quantum mechanics on the one hand and those of molecular chemistry on the other can be related in principle, Dirac's mathematical complications still remain as the derivational work necessary to patch the two domains together is mathematically difficult.

\section{Re-conceiving Reduction and Emergence}

So far I have argued that the relationship between quantum mechanics and molecular chemistry-principally regarding how molecular structure arises-is not well characterized by either typical reductionist or emergentist accounts. Or put another way, in the light of current practice in the physical sciences regarding quantum mechanics and molecular chemistry, popular ways of formulating reductionist and emergentist theses are either false or highly misleading. This situation suggests that re-conceiving the categories such as reduction and emergence might be fruitful and, taking cues from the practice of the physical sciences, I sketch the following alternative for the relevant categories (compare with Bishop and Atmanspacher submitted): 
Reduction

Contextual Emergence

Supervenience

Strong Emergence
"More fundamental" properties/descriptions provide NECESSARY and SUFFICIENT conditions for "less fundamental" properties/descriptions

"More fundamental" properties/descriptions provide NECESSARY but not sufficient conditions for "less fundamental" properties/descriptions

"More fundamental" properties/descriptions provide SUFFICIENT but not necessary conditions for "less fundamental" properties/descriptions

"More fundamental" properties/descriptions provide NEITHER necessary nor sufficient conditions for "less fundamental" properties/descriptions

With respect to this schema, the usual story regarding chemistry and physics would fall under reduction. Here, the relevant set of necessary and sufficient conditions are the fundamental particles of quantum mechanics, their properties and the quantum laws governing them. But, as I have argued, this approach to characterizing the relationship of molecular structure to quantum mechanics fails. On the other hand, the usual ways of characterizing property emergence sound very much like the last category in the schema-radical or strong emergence-except for the condition that emergent properties at some higher level arise out of the entities and properties at the lower level. If "arise out of" is construed in a reductionist fashion (e.g., Kim 1999), then we are back to reduction. On the other hand, if "arise out of" is to be taken as nonreductive, then it could be one of the other three categories depending on the details of the account. As pointed out above, however, standard way of characterizing emergent properties is highly misleading in the case of molecular structure. Rather, the relationship of quantum mechanics and molecular structure is best represented by the category of contextual emergence: quantum mechanics provides necessary conditions (e.g., in the form of electrons, nucleons, strong- and weak- nuclear forces, etc.), but provides no sufficient conditions (i.e., no molecular structures relating electrons and nucleons, their motions, etc.) for analyzing the relationship between quantum mechanics and molecular structure. Yet, with the appropriate contingent context, necessary and sufficient conditions relating quantum mechanics and molecular structure can be given, where the two domains overlap nontrivially.

There are, of course, other possible responses to the predicament represented in $\S \S 2$ and 3. One possibility is to simply sit tight and wait for the "final theory" of some future perfect physics to sort it all out. This is often a stock reductionist reply when all else fails. But aside from unwarranted fantasies of a future perfect or complete physics, this merely begs the question by hanging one's hope on a metaphysical intuition-about what a complete physics might mean regarding reductionism-rather than on argument.

Another possibility is to seek out an alternative to quantum mechanics. For example, one might turn to quantum field theory, but this has basically the same symmetry problems as quantum mechanics with respect to molecular structure. Or one might turn to rigged Hilbert space quantum mechanics (e.g., Bohm 1978). This generalization of quantum mechanics has the topological 
resources (e.g., it is not saddled with just the Hilbert space topology), but it is an open question as to whether it has the appropriate ontological resources.

Then there are what some might consider more radical possibilities like rejecting $\mathrm{CoPe}$ and/or CoPo. This perhaps amounts to some kind of emergence view, epistemological or ontological, and as such is consistent with contextual emergence. But it need not be too radical as, based on our best physical theories, there is no reason to suspect, aside from hidden reductionist metaphysical presuppositions and some misunderstandings of science, that physics should be exhaustive of the physical nor that physics should somehow be inert with respect to contexts set by chemistry and biology, say. ${ }^{2}$ And any downward causation that might arise from higher physical levels (e.g., chemistry) would certainly be consistent with the underlying physics on a contextual emergence view, since the physical laws forming part of the set of necessary and sufficient conditions for the higher chemical levels come from physics itself and are always operative albeit not in the sense that such laws exhaustively determine all events in a context-free manner. ${ }^{3}$

\section{Some Initial Objections}

One might object that Nagelian (Nagel 1961) and similar forms of reduction are actually stronger than reduction as conceptualized above. If the connectability condition read as an identity statement, then this might very well be the case because there is replacement of higher-level properties by lower-level ones rather than reduction. However, this reading of Nagel's connectability condition has been seriously challenged (e.g., by multiple realizability) and currently there is no general agreement on an alternative reading (e.g., nomic coexstensivity, or some form of contingent condition). Other possibilities for the connectability condition offered by Nagel are (1961, p. 354): (1) a set of logical connections, where the meaning of some term appearing in the higher-level theory (e.g., molecular structure) must be explicable in terms of the established meanings of theoretical primitives in the lower-level theory (e.g., elementary particles and their laws), or (2) the connections are physical hypotheses, "asserting that the occurrence of the state of affairs signified by a certain theoretical expression 'B' in [the lower-level theory, quantum mechanics, say ] is a sufficient (or necessary and sufficient) condition for the state of affairs designated by ' $\mathrm{A}$ ' [in the higher-level theory, molecular structure, say]." It appears, then, that the most one can make clear on a Nagelian account with these alternative construals of the connectability condition is that lower-level laws and properties provide necessary and sufficient conditions for the derivation of higher-level properties in some form; that is to say, Nagelian accounts are properly forms of reduction as characterized in $\S 4$.

It might also be objected that characterizing supervenience in terms of sufficient, but no necessary conditions is inadequate. A supervenience relation is generally understood as one of general covariance; i.e., if some property $\mathrm{Q}$ supervenes on $\mathrm{P}$, then there is no change in $\mathrm{Q}$ without a corresponding change in $\mathrm{P}$. Such a covariance relation entails that $\mathrm{P}$ is sufficient for $\mathrm{Q}$, but does not

${ }^{2}$ Furthermore, there are some good metaphysical reasons for rejecting either form of CoP (Crook and Gillett 2000; Gillett 2002).

${ }^{3}$ The idea that the laws of quantum mechanics should exhaustively determine all events, particularly those at the quantum level, in a context-free manner is closely related to atomistic physicalism. See $\$ 5$ below. 
preclude that it may also be necessary for Q as well. However, if the covariance relation we are trying to capture with the notion of supervenience requires necessary conditions as well, then it is no different in kind from a case of reduction (Kim 1998, 1999). The key (non-reductive) intuition of supervenience relies on sufficiency and mixing necessary and sufficient conditions for the covariance relation looks more like a category mistake than an objection to the proposed scheme.

One might also object that contextual emergence violates one of the core principles of atomistic physicalism as identified by, for example, Robert van Gulick: "The only law-like regularities needed for the determination of macro features by micro features are those that govern the interactions of those micro features in all contexts, systematic or otherwise" (2001, pg. 18). I take the force of such an objection would be that unless molecular structure is solely determined by the constituents and forces of quantum mechanics-independent of context-an important metaphysical principles (often supplying useful methodological guidance), is violated. However, if this principle of atomism is to be construed-as surely it often is-as implying reduction, i.e., that all properties and laws at the micro-level provide jointly necessary and sufficient conditions for determining macro features, then we have already seen that the example of molecular structure does not respect such a "metaphysical principle" with regards to quantum mechanics. Whatever contributions the laws and constituents of quantum mechanics make to molecular structure are conditional on contingent contexts. On the other hand, if the principle referred to by van Gulick is read as being consistent with the crucial role played by contingent contexts-i.e., not in a strong context-free way-then atomism would be cleaved from reduction. The thrust of this essay suggests that the latter possibility is the only viable one for preserving some form of atomism consistent with molecular chemistry.

Finally, one might object that the wrong lesson has been drawn from the example of molecular structure. The need for sophisticated mathematics (e.g., asymptotic analysis) should not be taken to imply anything about some kind of change in domain between quantum mechanics and molecular chemistry. Rather, it merely indicates the current state of our theoretical and mathematical knowledge and abilities, implying nothing about reductionism as an ontological thesis about the relationship between the two domains. However, although asymptotic analysis is indeed sophisticated and complex, this objection misses out the crucial point of asymptotic reasoning and the nature of the example in question. As noted above, molecular structure presupposes both a new topology and new ontology that are not given by quantum mechanics, but which are determined by the context (e.g., contingent symmetry-breaking features). It is definitely not the case that the sophisticated mathematics deployed is somehow obscuring the metaphysical issues. Rather, the metaphysical issues and the practices of science are driving the sophisticated mathematics in this example.

\section{Discussion}

The argument in favor of the schema of $\S 4$ in this essay is indirect in the sense that the example of molecular structure's relationship to quantum mechanics suggests that some typical ways people view reduction and emergence do not fit well with an important example from the physical sciences. Then, this example is used to support/illustrate the proposed schema. Although not done here, it should be possible to map most all accounts of reduction, supervenience and emergence into the schema, which is one of its advantages. A second advantage is that there are examples in physical science, such as molecular structure, that exhibit some features that somehow appear to sit in 
between typical reduction and emergence accounts, and the category of contextual emergence is able to capture these cases. ${ }^{4}$

The view I have sketched here differs from Nancy Cartwright's kind of patchwork view utilized in her arguments regarding realism, causation and modeling (e.g., 1994 and 1999) insofar as her view does not take into consideration the well-defined relations between different domains. Contextual emergence, as sketched in this essay, presupposes just such well defined relations among nontrivally overlapping domains (e.g., Primas 1998), which is one reason why it can form a viable alternative to typical accounts of reductive or emergent relations between domains.

One direction for further development of the proposed schema is to relate it to realization relations which have gained recent interest (e.g., Kim 1998, 1999; Crook and Gillett 2000; Gillett 2002). It would be quite illuminating to understand in more detail how the constituents in the quantum domain can act as realizers for the molecular structures in the domain of molecular chemistry absent sufficient conditions for such realizations in the former domain.

\section{Acknowledgments}

Early versions of this paper benefitted from presentations at the British Society for the Philosophy of Science Annual Meeting and the Popper Seminar in the Department of Philosophy, Logic and Scientific Method at the London School of Economics. Engaging discussions with Harald Atmanspacher, Fred Kronz, Carl Gillett and Hans Primas are gratefully acknowledged. The Alexander von Humboldt Foundation as well as the Federal Ministry of Education and Research and the Program for the Investment in the Future of the German Government provided much appreciated financial support.

\section{References}

Amann, A. (1993), "The Gestalt Problem in Quantum Theory: Generation of Molecular Shape by the Environment," Synthese 97: 125-156.

Avertisov, V., Goldanskii, V., and Kuz'min, V. (1991), "Handedness, Origin of Life and Evolution," Physics Today 44(2): 33-41.

Batterman, R. (2002), The Devil in the Details. Oxford: Oxford University Press.

Berry, M. (1994), 'Asymptotics, Singularities and the Reduction of Theories," in D. Prawitz,

B. Skyrms and D. Westerstahl (eds.),Logic, Methodology and Philosophy of Science

IX: Proceedings of the Ninth International Congress of Logic, Methodology and Philosophy of Science, Uppsala 1991. Amsterdam: Elsevier, North-Holland, pp. 597-607.

Bishop and Atmanspacher (submitted), "Contextual Emergence in the Description of Properties."

Bohm, A. (1978), The Rigged Hilbert Space and Quantum Mechanics, Lecture Notes in Physics Vol. 78, Springer, Berlin (1978).

Born, M., and Oppenheimer, R. (1927), “Zur Quantentheorie der Molekeln,” Annalen der Physik 84: 457-484.

${ }^{4}$ These kinds of "in between" cases are neither rare nor insignificant (e.g., Primas 1998; Bishop and Atmanspacher submitted). 
Cartwright, N. (1994), "Fundamentalism vs. the Patchwork of Laws," Proceedings of the Aristotelian Society 93: 279-282.

Cartwright, N. (1999), The Dappled World: A Study of the Boundaries of Science. Cambridge: Cambridge University Press.

Crook, S. and Gillett, C. (2001),"'Why Physics Alone Cannot Define the 'Physical',' Canadian Journal of Philosophy 31: 333-360.

DeCamp, W. H. (1989), "The FDA Perspective on the Development of Stereoisomers," Chirality 1: 2-6.

Dingle, R. (1973), Asymptotic Expansions: Their Derivation and Interpretation. New York: Academic Press.

Dirac, P. A. M. (1929), Quantum Mechanics of Many-Electron Systems," Proceedings of the Royal Society of London A123: 714-733.

Friedrichs, K. (1955), "Asymptotic Phenomena in Mathematical Physics," Bulletin of the American Mathematical Society 61: 485-504.

Gillett, C. (2002), "The Varieties of Emergence: Their Purposes, Obligations and Importance," Grazer Philosophische Studien 65: 95-121.

Gimzewski, J., and Joachim, C. (1999), "Nanoscale Science of Single Molecules Using Local Probes," Science 283: 1683-8.

Hendry, R. (1998), "Models and Approximations in Quantum Chemistry," in N. Shanks (ed.), Idealization IX: Idealization in Contemporary Physics, Pozna $\Omega$ Studies in the Philosophy of the Sciences and the Humanities 63. Amsterdam: Rodopi, pp. 123-142.

Kim, J. (1998), Mind in a Physical World: An Essay on the Mind-Body Problem and Mental Causation. Cambridge, MA: MIT Press.

Kim, J. (1999), “Making Sense of Emergence,” Philosophical Studies 95: 3-36.

Lehn, J.-M. (2002), “Toward Self-Organization and Complex Matter,” Science 295: 2400-2403.

Nagel, E. (1961), The Structure of Science. New York: Harcourt, Brace \& World.

Primas, H. (1983), Chemistry, Quantum Mechanics and Reductionism. Berlin: Springer.

Primas, H. (1998), "Emergence in Exact Natural Sciences," Acta Polytechnica Scandinavica 91: 83-98.

Smith, D., Babcock, H. and Chu, S. (1999), "Single-Polymer Dynamics in Steady Shear Flow," Science 283: 1724-1727.

Van Gulick, R. (2001), "Reduction, Emergence and Other Recent Options on the Mind/Body Problem," Journal of Consciousness Studies 8(9/10): 1-34.

Whitesides, G., and Grzybowski, B. (2002), "Self-Assembly at All Scales," Science 295: 2418-2421.

Weiss, S. (1999), "Fluorescence Spectroscopy of Single Biomolecules,” Science 283: 1676-1683.

Woolley, R. (1976), “Quantum Theory and Molecular Structure,” Advances in Physics 25: 27-52. 